\title{
Gossypiboma Presenting as an Atypical Intra-Abdominal Cyst: A Case Report
}

\author{
Dilip Dan, P. Ramraj, Viren Solomon, Olivier Leron, Malini Ramnarine, \\ Kavita Deonarine, Vijay Naraynsingh, Nigel Bascombe \\ Department of Surgery, University of the West Indies, St. Augustine, Trinidad and Tobago \\ Email: dilipdan5@gmail.com
}

Received 12 August 2014; revised 27 September 2014; accepted 13 October 2014

Copyright @ 2014 by authors and Scientific Research Publishing Inc.

This work is licensed under the Creative Commons Attribution International License (CC BY). http://creativecommons.org/licenses/by/4.0/

(c) (i) Open Access

\begin{abstract}
Gossypibomas are generally retained surgical sponges, and are usually a rare occurrence. They are diagnostic dilemmas with an incidence ranging from 1 in 8000 to 1 in 18,000 surgeries. However the incidence of this problem is on the rise and the clinician needs to have a high index of suspicion to make an accurate diagnosis. We reported the case of a 50-year-old male patient who presented with a 6-month history of vague epigastric discomfort, early satiety and nausea. Further investigation revealed an intra-abdominal cyst that proved to be secondary to a retained laparotomy sponge and was treated laparoscopically. This is usually an unanticipated surgical misadventure which is often preventable, with significant associated stigma for the surgical professional involved. Unfortunately it leads to extensive and often unnecessary surgical intervention. The condition can be managed conservatively or surgically. Our case report demonstrates the use of laparoscopy for the successful management of intra-abdominal gossypibomas and represents the first reported case of laparoscopic management of a gossypiboma in the Caribbean setting. It also demonstrates both the acute and delayed presentations of gossypibomas in the same patient.
\end{abstract}

\section{Keywords}

Gossypiboma, Intra-Abdominal Cyst, Laparoscopic

\section{Introduction}

Gossypibomas are generally retained surgical sponges, and are usually a rare occurrence. However the incidence of this problem is on the rise and the clinician needs to have a high index of suspicion to make an accurate diagnosis. We presented the case of an intra-abdominal cyst secondary to a retained laparotomy sponge which was treated laparoscopically.

How to cite this paper: Dan, D., Ramraj, P., Solomon, V., Leron, O., Ramnarine, M., Deonarine, K., Naraynsingh, V. and Bascombe, N. (2014) Gossypiboma Presenting as an Atypical Intra-Abdominal Cyst: A Case Report. Health, 6, 2499-2504. http://dx.doi.org/10.4236/health.2014.618287 


\section{Case Report}

This is the case of a 50-year-old male patient who presented with 6 month history of vague epigastric discomfort, early satiety and nausea. He had associated weight loss of approximately $10 \mathrm{~kg}$ over that period but there was no vomiting or change in bowel habit.

Of significance in his past surgical history was an open left sided nephrolithotomy for renal calculi approximately seven years previously. That admission was complicated by a reoperation one week after the index operation for failure of recovery and sepsis. A retained surgical swab was removed and he did well subsequently.

His abdominal examination revealed a vague mass in the left side of the abdomen causing mild abdominal asymmetry. This mass was approximately $15 \mathrm{~cm} \times 12 \mathrm{~cm}$. It was firm, smooth and non tender (Figure 1).

His hematological and biochemical parameters were all within normal limits. The patient went on to have a Computerized Tomography (CT) scan of the abdomen and pelvis which showed a large $21 \times 15 \mathrm{~cm}$ cystic lesion arising antero-inferiorly from the left kidney extending into the peritoneal cavity but non-communicating with the left kidney (Figure 2).

Gastroscopy and colonoscopy revealed that the mass was not arising or communicating with the upper or lower gastrointestinal tract. There were signs of external compression, but no mucosal lesion was visualized. A CT diagnosis of a mesenteric cyst was made and the patient was prepared for exploratory laparoscopy.

At operation the patient was placed under general anesthetic in the supine position. Laparoscopy was performed which showed a large $15 \mathrm{~cm} \times 20 \mathrm{~cm}$ left sided cystic lesion deep to the mesocolon and elevating it. The cyst was mobilized anteriorly and drained to facilitate access to its posterior aspect which seemed to be involved in an intense fibrotic reaction. The contents of the cyst were a turbid greenish color however subsequent culture showed no bacterial growth. At this point a decision was made to marsupialize the cyst. Once the cyst was deroofed it became evident that a foreign body was present within it (Figure 3).

The foreign body was identified as a laparotomy sponge and removed using a retrieval bag. A 15 French suction drain was left in situ. There was minimal post-operative pain. After two days the drainage was minimal and the patient discharged with an outpatient appointment the following week. On the seventh post-operative day the patient developed abdominal pains and high grade fever. A CT scan done at this time revealed an intra-abdominal abscess in the perinephric area and this was drained percutaneously (Figure 4). The patient recovered well subsequently.

\section{Discussion}

Gossypiboma refers to a retained surgical sponge and the associated foreign body reaction. Surgical sponges are the most frequently retained surgical foreign body accounting for approximately two thirds of retained objects [1]. They can range from small gauze pads to full towels. Small blood soaked sponges may be difficult to differ-

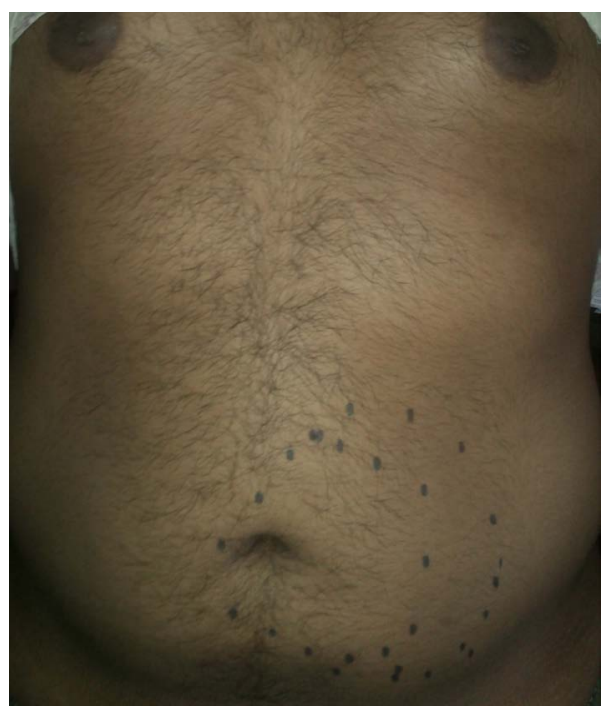

Figure 1. Asymmetrical mass in left mid abdomen highlighted by dotted outline. 


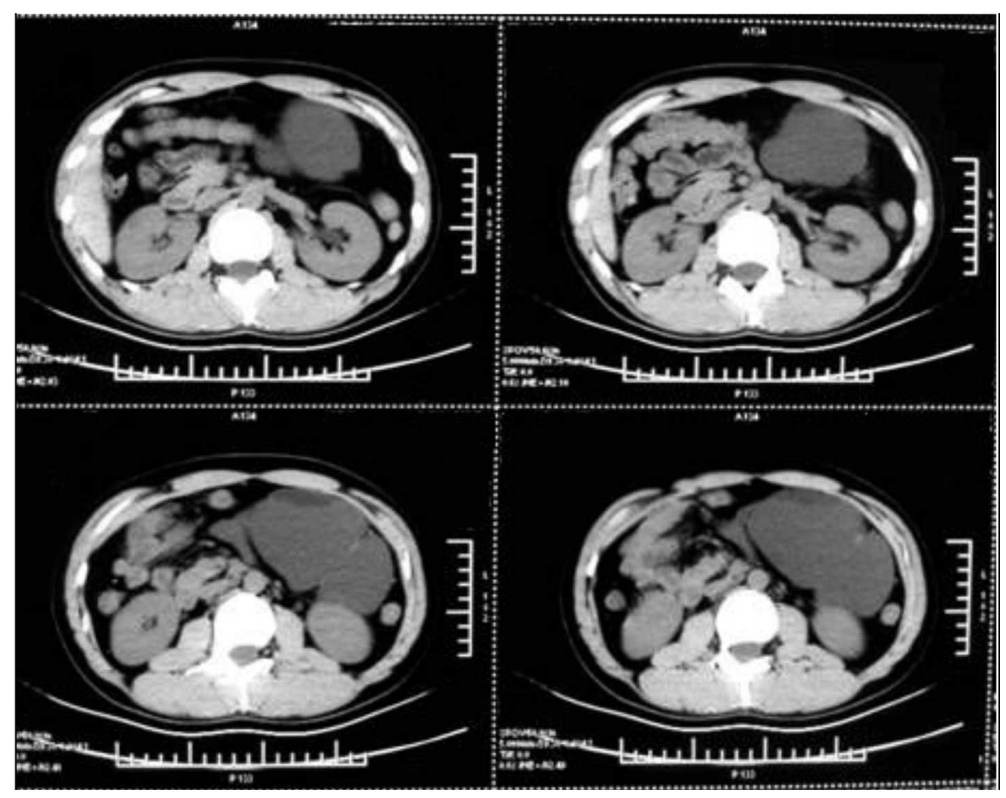

Figure 2. CT scan showing large cystic lesion abutting but separate from the left kidney. Composite non sequential images showing extent of cyst.

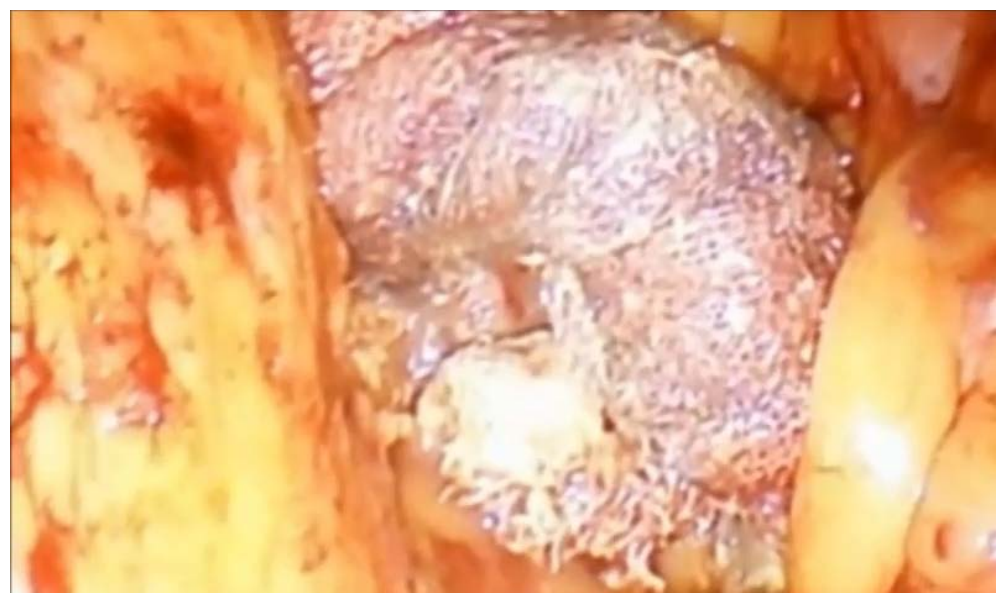

Figure 3. Gossypiboma located within cystic lesion in retroperitoneum.

rentiate from tissue and may be unintentionally left behind in a body cavity. The word has a dual origin; "gossypium" is the Latin word for cotton or wool and "boma" is a Kiswahili word for "place of concealment". It is also known as a textiloma, cottonoid or gauzeoma [2]. The incidence ranges from $1 / 8000$ to $1 / 18,000$ but this may be under reported due to the medico-legal implications [3].

Neurosurgical, gynecological, orthopedic, urological, vascular, neck exploration, maxillary sinus surgery, breast surgery and cardiothoracic surgical procedures have all been associated with the presence of gossypiboma. But by far it most commonly presents secondary to abdominal surgeries with an estimated incidence of 1/1000 to $1 / 1500$ [4]. This is due to the availability of large spaces for swabs to hide within the peritoneal cavity. It has even been reported in patients who have had laparoscopic surgery.

Certain physical characteristics like increasing body mass index and female gender may be associated with gossypiboma. Surgically complex procedures with longer operating times (more than four hours) requiring a change in assistive staff during the procedure and multiple procedures being done at the same time especially with multiple surgical teams [1] [5]. Human error is more likely to occur with inexperienced staff, during emergency procedures and when there is change in a planned procedure or complications [4].

A surgical sponge may lead to an exudative inflammatory process with abscess formation. This may present 


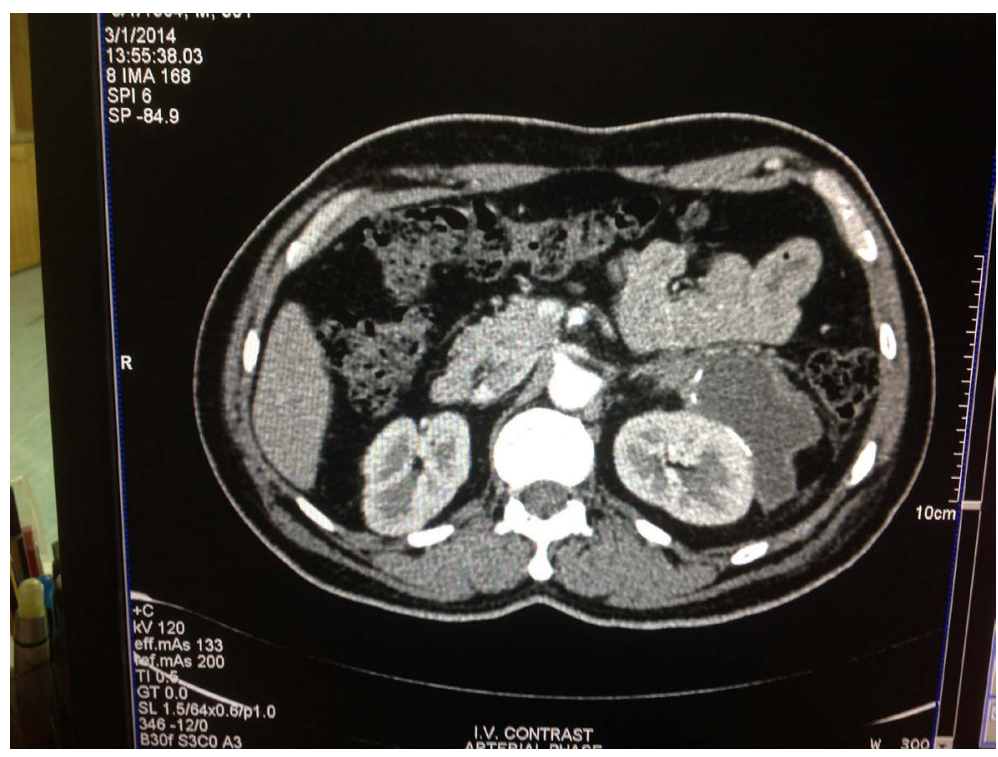

Figure 4. Post operative perinephric collection which was drained percutaneously via a posterior approach.

acutely in the early post-operative phase with sepsis leading to subsequent surgical removal (as in this patient in the index surgery). The more common form of presentation however is the patient who has had a chronic gossypiboma secondary to an aseptic fibrinous response with formation of adhesions and encapsulation leading to a walled off fibrinous mass as in our case. It can be picked up months or even years after initial surgery [6].

The 2009 World Health Organization guidelines for safe surgical procedures advocates only using radiopaque detectable sponges in body cavities [7]. Many hospitals have counting protocols that may include a swab count prior to the procedure, an interim count and a count after the procedure. Counts are audible and double checked by two nurses. However the propensity to err is intrinsic to human nature and evidence suggests that manual counting of sponges even under stringent protocols may be incorrect due to human error resulting in a retained sponge despite a reportedly normal count [8] [9].

Recommendations to prevent the occurrence of gossypiboma include a quiet organized operating theatre with minimal distractions allowing the surgeon and axillary staff the optimum conditions for concentrating. A meticulous sponge count should be done at the beginning and after closure [10]. Radiopaque sponges should be used in body cavities and a thorough exploration of the abdominal cavity should be done before closure [7]. If a discrepancy in swab count is detected a radiograph of the entire operating field should be done prior to closure [10].

Computerized automated sponge counting techniques and embedding sponges with a microchip or radiofrequency tag has been employed in some institutions to prevent retained sponges [10]-[12]. This may seem costly however a cost analysis showed that the cost of a retained sponge to an institution is approximately nine times the cost of sponge-counting technology [13].

Plain radiographs may show curved radio-opaque lines if radiopaque markers are present. Radiopaque densities with air spaces may be seen if a sponge is retained.

On ultrasound a gossypiboma may look like a well demarcated cystic or solid mass or have a combined appearance with an acoustic shadow [8]. The radiopaque marker may be present and show a wavy internal echogenic focus. However the radiopaque marker may disintegrate over time and may not be detected by radiographs or ultrasound. CT scanning is useful at this time. Sponges may look like a low density heterogeneous mass which may have a spongiform appearance secondary to trapped gas bubbles within the fibres of the sponge [14] [15]. Older lesions may have calcification in the wall or even rim enhancement which makes it difficult to differentiate from other intra-abdominal abscesses. Linear densities with peculiar whorled configuration may suggest a towel as the cause. Wavy striped high density areas may represent the radiopaque markers.

T1 weighted magnetic resonance imaging will typically reveal a low signal intensity mass and T2 may show a low signal intensity with high signal density whorled stripes in the centre. There may be poor gadolinium en- 
hancement and in some cases a serrated border to the inner wall [16]. Shiraev et al. describe a "row of dots" sign which is a linear row of dots on MRI representing an iatrogenic foreign body with foreign-body reaction specifically dressing gauze, due to its thick fibers and non-metallic nature [17].

With asymptomatic gossypibomas conservative management may be considered. However in symptomatic patient the mainstay of treatment is removal of the foreign body. This has been achieved by both laparoscopic and open methods. The use of laparoscopic surgery in the diagnosis and removal of gossypiboma has been reported in the literature but this is the first reported case in the Caribbean [18]-[20]. Adhesions may complicate gossypiboma removal and conversion to laparotomy may be necessary. The largest gossypiboma in the literature is reported as $20 \mathrm{~cm}$ by $20 \mathrm{~cm}$ which was removed by gastroscopy [21]. Our case was a $20 \mathrm{~cm}$ by $15 \mathrm{~cm}$ mass which was removed laparoscopically. Larger masses may require laparotomy.

Gossypibomas have a serious medicolegal and psychological impact on the patient, the surgeon and the institution. The patient may be incorrectly diagnosed with a sarcoma or recurrent tumour and may undergo emotional distress secondary to this diagnosis in addition to unnecessary staging procedures and surgical interventions. In cases where patients have bowel perforation or sub acute intestinal obstruction secondary to a sponge they may require an ostomy which can be debilitating and embarrassing. In addition there are risks associated with a second major operation, death being a distinct possibility. There are anecdotal stories of failure to fully disclose to the patient when a gossypiboma is detected incidentally or at surgery however this practice is not advocated.

There are many malpractice cases where the surgeon and the institution are found responsible for the retained surgical sponge despite the fact that surgeons may argue in their defense that they were told by the nurses that the sponge count was accurate. There are also cases where the institution alone was found accountable. However the patient always is compensated since the finding of a retained sponge is proof that negligence did occur.

Damage to reputation of surgeon and hospital may occur secondary to these lawsuits and contribute to the reluctance of surgeons to fully disclose to the patient. Surgeons who experience surgical error, feel guilt, shame and personal sense of blame as well as embarrassment among colleagues. They are more likely to have symptoms of depression which may be detrimental to their future work performance and overall well being [22] [23]. Surgical errors like gossypiboma are very complex issues and should be handled by the surgeon, patient care team, hospital safety committee and necessary insurance and legal stakeholders.

\section{Conclusion}

Retained surgical sponge (gossypiboma) is an uncommon finding though there may be under-reporting due to the sensitive nature. We presented a case of two gossypibomas in the same patient that presented in both the acute and then delayed formats. The first was immediately post-op with sepsis and the latter several years later with an abdominal cyst. The latter was managed laparoscopically with good results demonstrating the value of minimal invasive techniques in this condition. This report was unusual in that the same patient suffered twice as there was a second retained sponge despite an operation to remove one in the immediate post-op period of the index operation.

\section{References}

[1] Gawande, A.A., Studdert, D.M., Orav, E.J., Brennan, T.A. and Zinner, M.J. (2003) Risk Factors for Retained Instruments and Sponges after Surgery. New England Journal of Medicine, 348, 229-235. http://dx.doi.org/10.1056/NEJMsa021721

[2] Ulucay, T., Dizdar, M.G., Sunay Yavuz, M. and Asirdizer, M. (2010) The Importance of Medico-Legal Evaluation in a Case with Intraabdominal Gossypiboma. Forensic Science International, 198, e15-e18. http://dx.doi.org/10.1016/j.forsciint.2010.01.013

[3] McIntyre, L.K., Jurkovich, G.J., Gunn, M.L. and Maier, R.V. (2010) Gossypiboma: Tales of Lost Sponges and Lessons Learned. Archives of Surgery, 145, 770-775. http://dx.doi.org/10.1001/archsurg.2010.152

[4] Cima, R.R., Kollengode, A., Garnatz, J., Storsveen, A., Weisbrod, C. and Deschamps, C. (2008) Incidence and Characteristics of Potential and Actual Retained Foreign Object Events in Surgical Patients. Journal of the American College of Surgeons, 207, 80-87. http://dx.doi.org/10.1016/j.jamcollsurg.2007.12.047

[5] Wan, W., Le, T., Riskin, L. and Macario, A. (2009) Improving Safety in the Operating Room: A Systematic Literature Review of Retained Surgical Sponges. Current Opinion in Anaesthesiology, 22, 207-214. http://dx.doi.org/10.1097/ACO.0b013e328324f82d 
[6] Biswas, R.S., Ganguly, S., Saha, M.L., Saha, S., Mukherjee, S. and Ayaz, A. (2012) Gossypiboma and SurgeonCurrent Medicolegal Aspect-A Review. The Indian Journal of Surgery, 74, 318-322. http://dx.doi.org/10.1007/s12262-012-0446-3

[7] Organisation WHO (2009) WHO Guidelines for Safe Surgery: Safe Surgery Saves Lives.

[8] Zbar, A.P., Agrawal, A., Saeed, I.T. and Utidjian, M.R. (1998) Gossypiboma Revisited: A Case Report and Review of the Literature. Journal of the Royal College of Surgeons of Edinburgh, 43, 417-418.

[9] Greenberg, C.C., Regenbogen, S.E., Lipsitz, S.R., Diaz-Flores, R. and Gawande, A.A. (2008) The Frequency and Significance of Discrepancies in the Surgical Count. Annals of Surgery, 248, 337-341. http://dx.doi.org/10.1097/SLA.0b013e318181c9a3

[10] Imhof, M. (2012) Malpractice in Surgery: Safety Culture and Quality Managemnet in the Hospital: Walter de Gruyter. http://dx.doi.org/10.1515/9783110271607

[11] Greenberg, C.C., Diaz-Flores, R., Lipsitz, S.R., Regenbogen, S.E., Mulholland, L., Mearn, F., et al. (2008) Bar-Coding Surgical Sponges to Improve safety: A Randomized Controlled Trial. Annals of Surgery, 247, 612-616. http://dx.doi.org/10.1097/SLA.0b013e3181656cd5

[12] Cima, R.R., Kollengode, A., Clark, J., Pool, S., Weisbrod, C., Amstutz, G.J., et al. (2011) Using a Data-Matrix-Coded Sponge Counting System across a Surgical Practice: Impact after 18 Months. Joint Commission Journal on Quality and Patient Safety, 37, 51-58.

[13] Sloane, T. (2013) The High Cost of Inaction: Retained Surgical Sponges Are Draining Hospital Finances and Ruining Reputations. Becker's Infection Control \& Clinical Quality.

[14] Apter, S., Hertz, M., Rubinstein, Z.J. and Zissin, R. (1990) Gossypiboma in the Early Post-Operative Period: A Diagnostic Problem. Clinical Radiology, 42, 128-129. http://dx.doi.org/10.1016/S0009-9260(05)82084-7

[15] Moyle, H., Hines, O.J. and McFadden, D.W. (1996) Gossypiboma of the Abdomen. Archives of Surgery, 131, $566-568$. http://dx.doi.org/10.1001/archsurg.1996.01430170112022

[16] Kim, C.K., Park, B.K. and Ha, H. (2007) Gossypiboma in Abdomen and Pelvis: MRI Findings in Four Patients. American Journal of Roentgenology, 189, 814-817. http://dx.doi.org/10.2214/AJR.07.2323

[17] Shiraev, T., Bonar, S.F., Stalley, P. and Anderson, S.E. (2013) MRI "Row of Dots Sign” in Gossypiboma: An Enlarging Mass 8 Months after Sarcoma Resection. Skeletal Radiology, 42, 1017-1019. http://dx.doi.org/10.1007/s00256-013-1596-5

[18] Singh, R., Mathur, R.K., Patidar, S. and Tapkire, R. (2004) Gossypiboma: Its Laparoscopic Diagnosis and Removal. Surgical Laparoscopy, Endoscopy \& Percutaneous Techniques, 14, 304-305.

[19] Justo, J.W., Sandler, P. and Cavazzola, L.T. (2013) Retained Surgical Sponge Mimicking GIST: Laparoscopic Diagnosis and Removal 34 Years after Original Surgery. Journal of Minimal Access Surgery, 9, 29-30.

[20] Ramdass, M., Maharaj, D. and Naraynsingh, V. (2002) Gossypiboma: A Diagnostic Dilemma. The Internet Journal of Radiology, 2, 8.

[21] Sozutek, A., Yormaz, S., Kupeli, H. and Saban, B. (2013) Transgastric Migration of Gossypiboma Remedied with Endoscopic Removal: A Case Report. BMC Research Notes, 6, 413. http://dx.doi.org/10.1186/1756-0500-6-413

[22] Waterman, A.D., Garbutt, J., Hazel, E., Dunagan, W.C., Levinson, W., Fraser, V.J., et al. (2007) The Emotional Impact of Medical Errors on Practicing Physicians in the United States and Canada. Joint Commission Journal on Quality and Patient Safety, 33, 467-476.

[23] Balch, C.M., Oreskovich, M.R., Dyrbye, L.N., Colaiano, J.M., Satele, D.V., Sloan, J.A. and Shanafelt, T.D. (2011) Personal Consequences of Malpractice Lawsuits on American Surgeons. Journal of the American College of Surgeons, 213, 657-667. http://dx.doi.org/10.1016/j.jamcollsurg.2011.08.005 
Scientific Research Publishing (SCIRP) is one of the largest Open Access journal publishers. It is currently publishing more than 200 open access, online, peer-reviewed journals covering a wide range of academic disciplines. SCIRP serves the worldwide academic communities and contributes to the progress and application of science with its publication.

Other selected journals from SCIRP are listed as below. Submit your manuscript to us via either submit@scirp.org or Online Submission Portal.
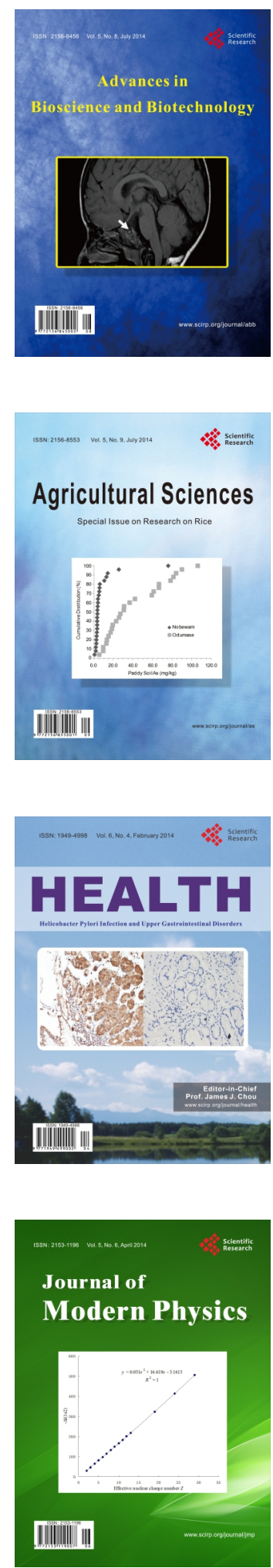
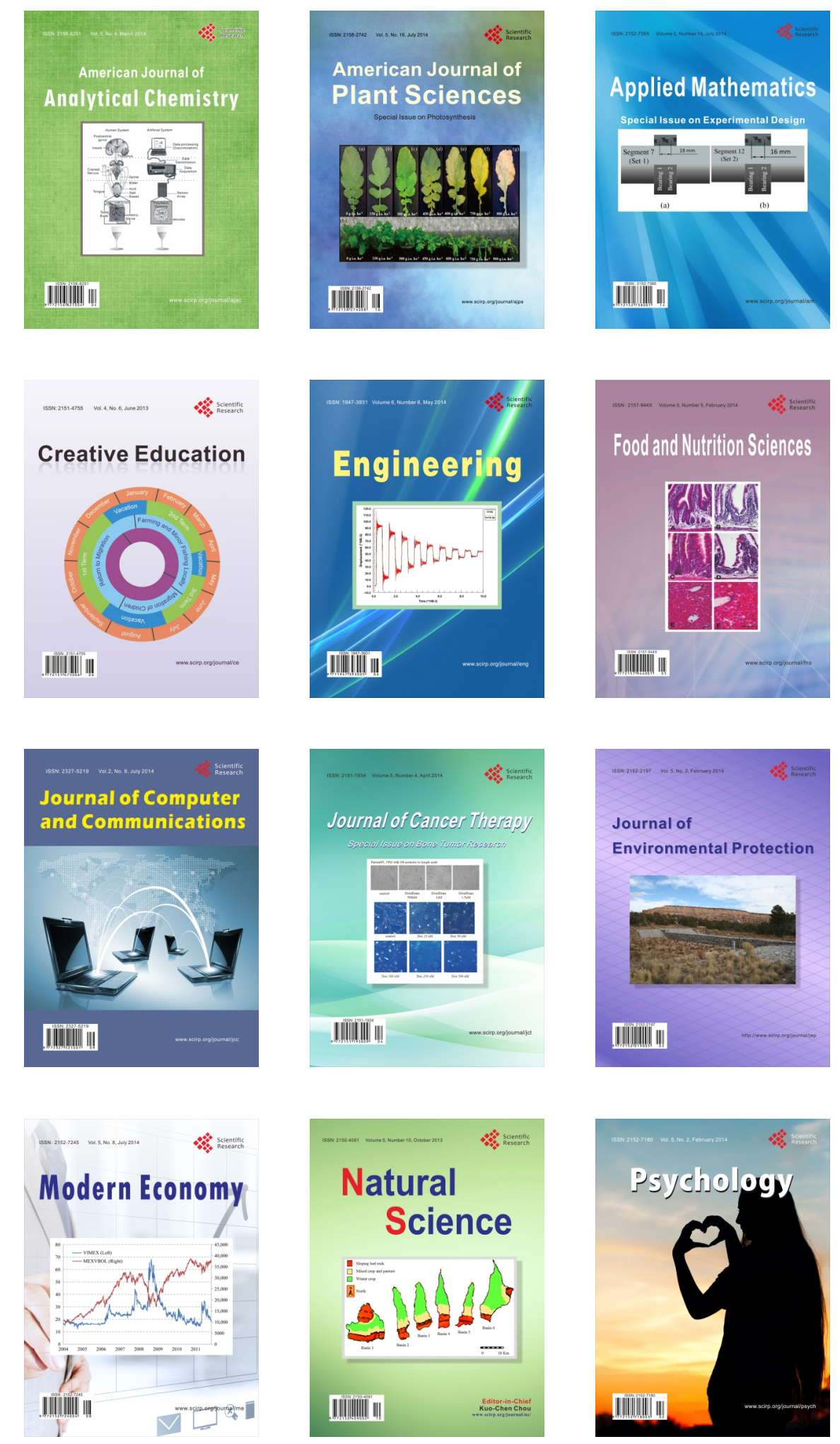